\title{
Symmetric properties of 2-D sequences and their applications for designing linear-phase 2-D FIR digital filters
}

\author{
Soo-Chang Pei ${ }^{\mathrm{a}, *}$, Jong-Jy Shyu ${ }^{\mathrm{b}}$ \\ ${ }^{2}$ Department of Electrical Engineering, National Taiwan University, Taipei, Taiwan, ROC \\ ${ }^{\mathrm{b}}$ Department of Computer Science and Engineering, Tatung Institute of Technology, Taipei, Taiwan, ROC
}

Reccived 22 April 1994

\begin{abstract}
Besides the design of quadrantally symmetric linear-phase 2-D filters, various linear-phase 2-D filter designs are proposed in this paper. We will start from the discussion of the symmetric properties of 2-D sequences to disclose their applications for designing linear-phase 2-D FIR digital filters. It is shown that there are 16 types of cases to be considered according to the symmetry/antisymmetry of 2-D sequences in both directions and their filter lengths (even or odd). The corresponding types of amplitude responses are tabulated into a complete table if these 2-D sequences are used to realize 2-D FIR filters. Also, the definition of quadrantal-plane, half-plane and full-plane filters are described along with numerical examples designed by the eigenfilter approach.
\end{abstract}

\section{Zusammenfassung}

Neben dem Entwurf von linearphasigen, bezüglich der Quadranten symmetrischen 2D-Filtern werden in dieser Arbeit verschicdenc Entwürfe linearphasiger 2D-Filter vorgeschlagen. Wir beginnen mit der Diskussion der Symmetrie-Eigenschaften von 2D-Folgen, um ihre Anwendung zum Entwurf von 2-D-FIR-Filtern zu verdeutlichen. Es wird gezeigt, daß sechzehn verschiedene Fälle bezüglich der Symmetrie/Antimetrie von 2D-Folgen in beide Richtungen und bezüglich ihrer Filterlängen (gerade oder ungerade) zu betrachten sind. Die zugehörigen Typen von Frequenzgängen bei Nutzung der 2D-Folgen zum Entwurf von 2D-Filtern werden tabellarisch dargestellt. Weiterhin werden die Definitionen von Quadranten-, Halb- und Vollebenen-Filtern angegeben und durch numerische Beispicle anhand von Eigenfilter-Lösungen voranschaulicht.

\section{Résumé}

Dans cet article, outre la conception de filtres 2D a phase linéaire symétriques par quadrant, on propose plusieurs conceptions de filtres 2D à phase linéaire. Nous commencerons par discuter les propriétés de symétrie des séquences 2D pour révéler leurs applications dans la conception de filtres numériques FIR 2D à phase linéaire. On montre qu'il existe 16 cas-types a considérer, d'après la symétrie/disymétrie des séquences $2 \mathrm{D}$ dans les deux directions et la longueur de leurs filtres (pair ou impair). Les types de réponse d'amplitude correspondants sont placés dans un tableau complet si ces séquences 2D sont utilisées pour réaliser des filtres FIR 2D. De même, on trouvera les définitions des filtres quadrant. demi-plan et plan, accompagnés d'exemples numériques calculés par l'approche des filtres propres.

Keywords: Filter symmetry; 2-D FIR filter; Filter design

* Corresponding author. 


\section{Introduction}

Conventionally, the design of linear-phase 2-D FIR digital filters is concentrated on the class of quadrantally symmetric filters, such as circular filters, fan-type filters, etc. [1]. A 2-D sequence, which is symmetric in both directions, is required to realize such quadrantally symmetric filters.

In this paper, we will start from the discussion of the symmetric properties of 2-D sequences to disclose their applications for designing linear-phase 2-D FIR digital filters by the eigenfilter approach, which has been used successfully to design linearphase 1-D filters $[2,3]$ and 2-D quadrantally symmetric filters [4]. It is shown that there are 16 types of cases to be considered according to the symmetry/antisymmetry of 2-D sequences in both directions and their filter lengths (even or odd). The corresponding types of amplitude responses are tabulated into a complete table if these 2-D sequences are used to realize 2-D FIR filters. Also, the definitions of quadrantal-plane, half-plane and full-plane filters are described along with several numerical design examples.

\section{Symmetric properties of $2-D$ sequences}

Let $X$ represent an $N_{1} \times N_{2}$ 2-D sequence in matrix form with its elements being denoted by $x\left(n_{1}, n_{2}\right)$, $n_{1}=0,1, \ldots, N_{1}-1, n_{2}=0,1, \ldots, N_{2}-1$, i.e. the sequence is called an odd-symmetric 2-D sequence in the $n_{1}$-direction; similarly for the $n_{2}$-direction. Then symmetric or antisymmetric 2-D sequences can be divided into four major types.

Type I: Even symmetry in both the $n_{1}$ - and $n_{2}$-directions, i.e.

$$
\begin{aligned}
x\left(n_{1}, n_{2}\right) & =x\left(N_{1}-1-n_{1}, n_{2}\right) \\
& =x\left(n_{1}, N_{2}-1-n_{2}\right), \\
0 \leqslant n_{1} & \leqslant N_{1}-1,0 \leqslant n_{2} \leqslant N_{2}-1 .
\end{aligned}
$$

Such an even-even sequence is denoted by $\boldsymbol{X}_{\mathrm{ee}}$.

Type II: Even symmetry in the $n_{1}$-direction and odd symmetry in the $n_{2}$-direction, i.e.

$$
\begin{aligned}
x\left(n_{1}, n_{2}\right) & =x\left(N_{1}-1-n_{1}, n_{2}\right) \\
& =-x\left(n_{1}, N_{2}-1-n_{2}\right), \\
0 \leqslant n_{1} & \leqslant N_{1}-1,0 \leqslant n_{2} \leqslant N_{2}-1 .
\end{aligned}
$$

We denote such an even-odd sequence by $\boldsymbol{X}_{\mathrm{eo}}$.

Type III: Odd symmetry in the $n_{1}$-direction and even symmetry in the $n_{2}$-direction, i.e.

$$
\begin{aligned}
x\left(n_{1}, n_{2}\right) & =-x\left(N_{1}-1-n_{1}, n_{2}\right) \\
& =x\left(n_{1}, N_{2}-1-n_{2}\right), \\
0 \leqslant n_{1} & \leqslant N_{1}-1,0 \leqslant n_{2} \leqslant N_{2}-1 .
\end{aligned}
$$

We denote such an odd-even sequence by $\boldsymbol{X}_{\mathbf{o e}}$.

$$
\boldsymbol{X}=\left[\begin{array}{cccc}
x(0,0) & x(0,1) & \cdots & x\left(0, N_{2}-1\right) \\
x(1,0) & x(1,1) & \cdots & x\left(1, N_{2}-1\right) \\
\vdots & \vdots & \ddots & \vdots \\
x\left(N_{1}-1,0\right) & x\left(N_{1}-1,1\right) & \cdots & x\left(N_{1}-1, N_{2}-1\right)
\end{array}\right]
$$

If

$$
\begin{aligned}
& x\left(n_{1}, n_{2}\right)=x\left(N_{1}-1-n_{1}, n_{2}\right), \quad 0 \leqslant n_{1} \leqslant N_{1}-1, \\
& 0 \leqslant n_{2} \leqslant N_{2}-1,
\end{aligned}
$$

we call $\boldsymbol{X}$ an even-symmetric $2-\mathrm{D}$ sequence in the $n_{1}$-direction, and if

$$
\begin{aligned}
& x\left(n_{1}, n_{2}\right)=-x\left(N_{1}-1-n_{1}, n_{2}\right), \\
& 0 \leqslant n_{1} \leqslant N_{1}-1,0 \leqslant n_{2} \leqslant N_{2}-1,
\end{aligned}
$$

Type IV: Odd symmetry in both the $n_{1}$ - and $n_{2}$-directions, i.e.

$$
\begin{aligned}
x\left(n_{1}, n_{2}\right) & =-x\left(N_{1}-1-n_{1}, n_{2}\right) \\
& =-x\left(n_{1}, N_{2}-1-n_{2}\right), \\
0 \leqslant n_{1} & \leqslant N_{1}-1,0 \leqslant n_{2} \leqslant N_{2}-1 .
\end{aligned}
$$

We denote such an odd-odd sequence by $\boldsymbol{X}_{0 o}$. 
For any $N_{1} \times N_{2}$ 2-D sequence $X$, it can always be decomposed into the above four types of $2-D$ sequences, i.e.

$\boldsymbol{X}=\boldsymbol{X}_{\mathrm{ee}}+\boldsymbol{X}_{\mathrm{eo}}+\boldsymbol{X}_{\mathrm{oc}}+\boldsymbol{X}_{\mathrm{oo}}$

and $\boldsymbol{X}_{\mathrm{ee}}, \boldsymbol{X}_{\mathrm{eo}}, \boldsymbol{X}_{\mathrm{oe}}$ and $\boldsymbol{X}_{\mathrm{oo}}$ can be calculated from $X$ by

$$
\begin{aligned}
& x_{\text {ee }}\left(n_{1}, n_{2}\right)=\frac{1}{4}\left[x\left(n_{1}, n_{2}\right)+x\left(N_{1}-1-n_{1}, n_{2}\right)\right. \\
& +x\left(n_{1}, N_{2}-1-n_{2}\right) \\
& \left.+x\left(N_{1}-1-n_{1}, N_{2}-1-n_{2}\right)\right] \text {, } \\
& 0 \leqslant n_{1} \leqslant N_{1}-1,0 \leqslant n_{2} \leqslant N_{2}-1 \text {, } \\
& x_{\mathrm{eo}}\left(n_{1}, n_{2}\right)=\frac{1}{4}\left[x\left(n_{1}, n_{2}\right)+x\left(N_{1}-1-n_{1}, n_{2}\right)\right. \\
& -x\left(n_{1}, N_{2}-1-n_{2}\right) \\
& \left.-x\left(N_{1}-1-n_{1}, N_{2}-1-n_{2}\right)\right] \text {, } \\
& 0 \leqslant n_{1} \leqslant N_{1}-1,0 \leqslant n_{2} \leqslant N_{2}-1 \text {, } \\
& x_{\mathrm{oc}}\left(n_{1}, n_{2}\right)=\frac{1}{4}\left[x\left(n_{1}, n_{2}\right)-x\left(N_{1}-1-n_{1}, n_{2}\right)\right. \\
& +x\left(n_{1}, N_{2}-1-n_{2}\right) \\
& \left.-x\left(N_{1}-1-n_{1}, N_{2}-1-n_{2}\right)\right] \text {, } \\
& 0 \leqslant n_{1} \leqslant N_{1}-1,0 \leqslant n_{2} \leqslant N_{2}-1
\end{aligned}
$$

and

$$
\begin{aligned}
x_{\mathrm{oo}}\left(n_{1}, n_{2}\right)=\frac{1}{4}[ & x\left(n_{1}, n_{2}\right)-x\left(N_{1}-1-n_{1}, n_{2}\right) \\
& -x\left(n_{1}, N_{2}-1-n_{2}\right) \\
& \left.+x\left(N_{1}-1-n_{1}, N_{2}-1-n_{2}\right)\right], \\
0 \leqslant n_{1} \leqslant N_{1} & -1,0 \leqslant n_{2} \leqslant N_{2}-1,
\end{aligned}
$$

where $x_{\mathrm{ee}}\left(n_{1}, n_{2}\right), \quad x_{\mathrm{eo}}\left(n_{1}, n_{2}\right), \quad x_{\mathrm{oe}}\left(n_{1}, n_{2}\right)$ and $x_{\mathrm{oo}}\left(n_{1}, n_{2}\right)$ are the elements of $\boldsymbol{X}_{\mathrm{ee}}, \boldsymbol{X}_{\mathrm{eo}}, \boldsymbol{X}_{\mathrm{oe}}$ and $\boldsymbol{X}_{\mathrm{oo}}$, respectively. We can also find that

$\boldsymbol{X}_{\mathrm{e}}=\boldsymbol{X}_{\mathrm{ee}}+\boldsymbol{X}_{\mathrm{oo}}$

and

$\boldsymbol{X}_{\mathrm{o}}=\boldsymbol{X}_{\mathrm{eo}}+\boldsymbol{X}_{\mathrm{oe}}$,

where $\boldsymbol{X}_{\mathrm{e}}$ is a centro even-symmetric 2-D sequence whose elements satisfy

$$
\begin{gathered}
x_{\mathrm{e}}\left(n_{1}, n_{2}\right)=x_{\mathrm{c}}\left(N_{1}-1-n_{1}, N_{2}-1-n_{2}\right), \\
0 \leqslant n_{1} \leqslant N_{1}-1,0 \leqslant n_{2} \leqslant N_{2}-1,
\end{gathered}
$$

and where $\boldsymbol{X}_{\mathrm{o}}$ is a centro odd-symmetric 2-D sequence whose elements satisfy

$$
\begin{gathered}
x_{\mathrm{o}}\left(n_{1}, n_{2}\right)=-x_{\mathrm{o}}\left(N_{1}-1-n_{1}, N_{2}-1-n_{2}\right), \\
0 \leqslant n_{1} \leqslant N_{1}-1,0 \leqslant n_{2} \leqslant N_{2}-1
\end{gathered}
$$

For example, if $\boldsymbol{X}$ is given by

$$
X=n_{1}\left[\begin{array}{rrrr}
8 & 0 & 8 & 0 \\
3 & 6 & -10 & -5 \\
11 & -8 & -8 & 7 \\
-2 & -6 & 10 & 2
\end{array}\right] \text {, }
$$

then

$$
\begin{aligned}
& \boldsymbol{X}_{\mathrm{ec}}=n_{1}\left[\begin{array}{rrrr}
2 & 3 & 3 & 2 \\
4 & -5 & -5 & 4 \\
4 & -5 & -5 & 4 \\
2 & 3 & 3 & 2
\end{array}\right], \\
& \boldsymbol{X}_{\mathrm{eo}}=n_{1}\left[\begin{array}{rrrr}
1 & -6 & 6 & -1 \\
3 & 4 & -4 & -3 \\
3 & 4 & -4 & -3 \\
1 & -6 & 6 & -1
\end{array}\right],
\end{aligned}
$$

$$
\boldsymbol{X}_{\mathrm{oe}}=n_{1}\left[\begin{array}{rrrr}
2 & 1 & 1 & 2 \\
-5 & 3 & 3 & -5 \\
5 & -3 & -3 & 5 \\
-2 & -1 & -1 & -2
\end{array}\right]
$$

$$
\left.X_{\mathrm{oo}}=n_{1} n_{2} \text { [rrrr} \begin{array}{rrrr}
3 & 2 & -2 & -3 \\
1 & 4 & -4 & -1 \\
-1 & -4 & 4 & 1 \\
-3 & -2 & 2 & 3
\end{array}\right] \text {, }
$$


and

$$
\begin{aligned}
& X_{\mathrm{e}}=n_{1}\left[\begin{array}{rrrr}
5 & 5 & 1 & -1 \\
5 & -1 & -9 & 3 \\
3 & -9 & -1 & 5 \\
-1 & 1 & 5 & 5
\end{array}\right], \\
& X_{\mathrm{o}}=n_{1}\left[\begin{array}{rrrr}
3 & -5 & 7 & 1 \\
-2 & 7 & -1 & -8 \\
8 & 1 & -7 & 2 \\
-1 & -7 & 5 & -3
\end{array}\right] .
\end{aligned}
$$

\section{Properties of frequency responses of 2-D sequences for designing linear-phase FIR digital filters}

The frequency response of a 2-D FIR digital filter with its impulse response $h\left(n_{1}, n_{2}\right), n_{1}=0,1, \ldots$, $N_{1}-1, n_{2}=0,1, \ldots, N_{2}-1$, can be characterized as

$$
H\left(\omega_{1}, \omega_{2}\right)=\sum_{n_{1}=0}^{N_{1}-1} \sum_{n_{2}=0}^{N_{2}-1} h\left(n_{1}, n_{2}\right) \mathrm{e}^{-\mathrm{j} n_{1} \omega_{1}} \mathrm{e}^{-\mathrm{j} n_{2}\left(\omega_{2}\right.} .
$$

If $h\left(n_{1}, n_{2}\right)$ is one of the four types of 2-D sequences, Eq. (24) can be rewritten as

$$
\begin{aligned}
H\left(\omega_{1}, \omega_{2}\right)= & \exp \left(-\mathrm{j} \frac{N_{1}-1}{2} \omega_{1}\right) \\
& \times \exp \left(-\mathrm{j} \frac{N_{2}-1}{2} \omega_{2}\right) \\
& \times \exp \left(\mathrm{j} \frac{M \pi}{2}\right) \hat{H}\left(\omega_{1}, \omega_{2}\right),
\end{aligned}
$$

where

$$
M= \begin{cases}0, & \text { Type I, } \\ 1, & \text { Type II and Type III, } \\ 2, & \text { Type IV, }\end{cases}
$$

and $\hat{H}\left(\omega_{1}, \omega_{2}\right)$ is a real-valued function. Notice that by excluding the linear-phase part in (25), the frequency responses are real-valued functions for
Type I and Type IV sequences, and are imaginaryvalued functions for Type II and Type III sequences. For example, if $h\left(n_{1}, n_{2}\right)$ is a Type I 2-D sequence and $N_{1}, N_{2}$ are odd integers, then

$$
\begin{aligned}
\hat{H} & \left(\omega_{1}, \omega_{2}\right) \\
& =\sum_{n_{1}=0}^{\frac{1}{2}\left(N_{2}-1\right)} \sum_{n_{2}=0}^{\frac{1}{2}\left(N_{2}-1\right)} a\left(n_{1}, n_{2}\right) \cos \left(n_{1} \omega_{1}\right) \cos \left(n_{2} \omega_{2}\right),
\end{aligned}
$$

which is a real-valued function and $a\left(n_{1}, n_{2}\right)$ are related to $h\left(n_{1}, n_{2}\right)$ by

$$
\begin{aligned}
& a(0,0)=h\left(\frac{N_{1}-1}{2}, \frac{N_{2}-1}{2}\right), \\
& a\left(0, n_{2}\right)=2 h\left(\frac{N_{1}-1}{2}, \frac{N_{2}-1}{2}-n_{2}\right), \\
& n_{2}=1, \ldots, \frac{N_{2}-1}{2},
\end{aligned}
$$

$$
\begin{gathered}
a\left(n_{1}, 0\right)=2 h\left(\frac{N_{1}-1}{2}-n_{1}, \frac{N_{2}-1}{2}\right), \\
n_{1}=1, \ldots, \frac{N_{1}-1}{2}, \\
a\left(n_{1}, n_{2}\right)=4 h\left(\frac{N_{1}-1}{2}-n_{1}, \frac{N_{2}-1}{2}-n_{2}\right), \\
n_{1}=1, \ldots, \frac{N_{1}-1}{2}, n_{2}=1, \ldots, \frac{N_{2}-1}{2} .
\end{gathered}
$$

Therefore, according to the four types of 2-D sequences discussed above and their even/odd lengths $\left(N_{1} \times N_{2}\right)$, there are 16 different kinds of $\hat{H}\left(\omega_{1}, \omega_{2}\right)$ which are tabulated in Table 1 . The relationships between the coefficients $a\left(n_{1}, n_{2}\right)$ 's in $\hat{H}\left(\omega_{1}, \omega_{2}\right)$ and $h\left(n_{1}, n_{2}\right)$ are listed in Table 2 .

As in the spatial-domain case, any magnitude response $\hat{H}\left(\omega_{1}, \omega_{2}\right)$ can be similarly decomposed into four parts in frequency domain as below:

$$
\begin{aligned}
\hat{H}\left(\omega_{1}, \omega_{2}\right)= & \hat{H}_{\mathrm{ee}}\left(\omega_{1}, \omega_{2}\right)+\hat{H}_{\mathrm{eo}}\left(\omega_{1}, \omega_{2}\right) \\
& +\hat{H}_{\mathrm{oe}}\left(\omega_{1}, \omega_{2}\right)+\hat{H}_{\mathrm{oo}}\left(\omega_{1}, \omega_{2}\right),
\end{aligned}
$$

where

$$
\begin{aligned}
\hat{H}_{\mathrm{ce}}\left(\omega_{1}, \omega_{2}\right) & =\hat{H}_{\mathrm{ec}}\left(-\omega_{1}, \omega_{2}\right)=\hat{H}_{\mathrm{ec}}\left(\omega_{1},-\omega_{2}\right) \\
& =\hat{H}_{\mathrm{ee}}\left(-\omega_{1},-\omega_{2}\right),
\end{aligned}
$$


Table 1

$\hat{H}\left(\omega_{1}, \omega_{2}\right)$ of 2-D sequences with length $N_{1} \times N_{2}\left(L_{i}=\frac{1}{2}\left(N_{i}-1\right)\right.$ for odd $N_{i}$ and $L_{i}=\frac{1}{2} N_{i}$ for even $\left.N_{i}, i=1,2\right)$

\begin{tabular}{|c|c|c|c|}
\hline Type & Sub-type & $N_{1}, N_{2}$ & $\hat{H}\left(\omega_{1}, \omega_{2}\right)$ \\
\hline \multirow[t]{4}{*}{1} & 1 & $N_{1}:$ odd, $N_{2}:$ odd & $\sum_{n_{1}=0}^{L_{1}} \sum_{n_{2}=0}^{L_{2}} a\left(n_{1}, n_{2}\right) \cos \left(n_{1} \omega_{1}\right) \cos \left(n_{2} \omega_{2}\right)$ \\
\hline & 2 & $N_{1}:$ odd, $N_{2}:$ even & $\sum_{n=0}^{L_{1}} \sum_{n_{2}=1}^{L_{2}} a\left(n_{1}, n_{2}\right) \cos \left(n_{1} \omega_{1}\right) \cos \left(\left(n_{2}-\frac{1}{2}\right) \omega_{2}\right)$ \\
\hline & 3 & $N_{1}:$ even, $N_{2}:$ odd & $\sum_{n_{1}=1}^{L_{1}} \sum_{n_{2}=0}^{L_{2}} a\left(n_{1}, n_{2}\right) \cos \left(\left(n_{1}-\frac{1}{2}\right) \omega_{1}\right) \cos \left(n_{2} \omega_{2}\right)$ \\
\hline & 4 & $N_{1}:$ even, $N_{2}:$ even & $\sum_{n_{1}=1}^{L_{1}} \sum_{n_{2}=1}^{L_{2}} a\left(n_{1}, n_{2}\right) \cos \left(\left(n_{1}-\frac{1}{2}\right) \omega_{1}\right) \cos \left(\left(n_{2}-\frac{1}{2}\right) \omega_{2}\right)$ \\
\hline \multirow[t]{4}{*}{ II } & 1 & $N_{1}:$ odd, $N_{2}:$ odd & $\sum_{n_{1}=0}^{L_{1}} \sum_{n_{2}=1}^{L_{2}} a\left(n_{1}, n_{2}\right) \cos \left(n_{1} \omega_{1}\right) \sin \left(n_{2} \omega_{2}\right)$ \\
\hline & 2 & $N_{1}:$ odd, $N_{2}:$ even & $\sum_{n_{1}=0}^{L_{1}} \sum_{n_{2}=1}^{L_{2}} a\left(n_{1}, n_{2}\right) \cos \left(n_{1} \omega_{1}\right) \sin \left(\left(n_{2}-\frac{1}{2}\right) \omega_{2}\right)$ \\
\hline & 3 & $N_{1}:$ even, $N_{2}:$ odd & $\sum_{n_{1}=1}^{L_{2}} \sum_{n_{2}=1}^{L_{2}} a\left(n_{1}, n_{2}\right) \cos \left(\left(n_{1}-\frac{1}{2}\right) \omega_{1}\right) \sin \left(n_{2} \omega_{2}\right)$ \\
\hline & 4 & $N_{1}$ : even, $N_{2}$ : even & $\sum_{n_{1}=1}^{L_{1}} \sum_{n_{2}=1}^{L_{2}} a\left(n_{1}, n_{2}\right) \cos \left(\left(n_{1}-\frac{1}{2}\right) \omega_{1}\right) \sin \left(\left(n_{2}-\frac{1}{2}\right) \omega_{2}\right)$ \\
\hline \multirow[t]{4}{*}{ III } & 1 & $N_{1}:$ odd, $N_{2}:$ odd & $\sum_{n_{1}=1}^{L_{1}} \sum_{n_{2}=0}^{L_{2}} a\left(n_{1}, n_{2}\right) \sin \left(n_{1} \omega_{1}\right) \cos \left(n_{2} \omega_{2}\right)$ \\
\hline & 2 & $N_{1}:$ odd, $N_{2}:$ even & $\sum_{n_{1}=1}^{L_{1}} \sum_{n_{2}=1}^{L_{2}} a\left(n_{1}, n_{2}\right) \sin \left(n_{1} \omega_{1}\right) \cos \left(\left(n_{2}-\frac{1}{2}\right) \omega_{2}\right)$ \\
\hline & 3 & $N_{1}:$ even, $N_{2}:$ odd & $\sum_{n_{1}=1}^{L_{1}} \sum_{n_{2}=0}^{L_{2}} a\left(n_{1}, n_{2}\right) \sin \left(\left(n_{1}-\frac{1}{2}\right) \omega_{1}\right) \cos \left(n_{2} \omega_{2}\right)$ \\
\hline & 4 & $N_{1}$ : even, $N_{2}$ : even & $\sum_{n_{1}=1}^{L_{1}} \sum_{n_{2}=1}^{L_{2}} a\left(n_{1}, n_{2}\right) \sin \left(\left(n_{1}-\frac{1}{2}\right) \omega_{1}\right) \cos \left(\left(n_{2}-\frac{1}{2}\right) \omega_{2}\right)$ \\
\hline \multirow[t]{4}{*}{ IV } & 1 & $N_{1}:$ odd, $N_{2}:$ odd & $\sum_{n_{1}=1}^{L_{1}} \sum_{n_{2}=1}^{L_{2}} a\left(n_{1}, n_{2}\right) \sin \left(n_{1} \omega_{1}\right) \sin \left(n_{2} \omega_{2}\right)$ \\
\hline & 2 & $N_{1}:$ odd, $N_{2}:$ even & $\sum_{n_{1}=1}^{L_{1}} \sum_{n_{2}=1}^{L_{2}} a\left(n_{1}, n_{2}\right) \sin \left(n_{1} \omega_{1}\right) \sin \left(\left(n_{2}-\frac{1}{2}\right) \omega_{2}\right)$ \\
\hline & 3 & $N_{1}:$ even, $N_{2}:$ odd & $\sum_{n_{1}=1}^{L_{1}} \sum_{n_{2}=1}^{L_{2}} a\left(n_{1}, n_{2}\right) \sin \left(\left(n_{1}-\frac{1}{2}\right) \omega_{1}\right) \sin \left(n_{2} \omega_{2}\right)$ \\
\hline & 4 & $N_{1}:$ even, $N_{2}:$ even & $\sum_{n_{1}=1}^{L_{1}} \sum_{n_{2}=1}^{L_{2}} a\left(n_{1}, n_{2}\right) \sin \left(\left(n_{1}-\frac{1}{2}\right) \omega_{1}\right) \sin \left(\left(n_{2}-\frac{1}{2}\right) \omega_{2}\right)$ \\
\hline
\end{tabular}

$$
\begin{aligned}
\hat{H}_{\mathrm{eo}}\left(\omega_{1}, \omega_{2}\right) & =\hat{H}_{\mathrm{eo}}\left(-\omega_{1}, \omega_{2}\right)=-\hat{H}_{\mathrm{eo}}\left(\omega_{1},-\omega_{2}\right) \\
& =-\hat{H}_{\mathrm{eo}}\left(-\omega_{1},-\omega_{2}\right) \\
\hat{H}_{\mathrm{oe}}\left(\omega_{1}, \omega_{2}\right) & =-\hat{H}_{\mathrm{oe}}\left(-\omega_{1}, \omega_{2}\right)=\hat{H}_{\mathrm{oe}}\left(\omega_{1},-\omega_{2}\right) \\
& =-\hat{H}_{\mathrm{oe}}\left(-\omega_{1},-\omega_{2}\right)
\end{aligned}
$$

and

$$
\begin{aligned}
\hat{H}_{\mathrm{oo}}\left(\omega_{1}, \omega_{2}\right) & =-\hat{H}_{\mathrm{oo}}\left(-\omega_{1}, \omega_{2}\right)=-\hat{H}_{\mathrm{oo}}\left(\omega_{1},-\omega_{2}\right) \\
& =\hat{H}_{\mathrm{oo}}\left(-\omega_{1},-\omega_{2}\right) .
\end{aligned}
$$


Table 2

Relationship between $a\left(n_{1}, n_{2}\right)$ in $\hat{H}\left(\omega_{1}, \omega_{2}\right)$ and $h\left(n_{1}, n_{2}\right)$ in $H\left(\omega_{1}, \omega_{2}\right)$

\begin{tabular}{|c|c|}
\hline Type & Relationship between $a\left(n_{1}, n_{2}\right)$ and $h\left(n_{1}, n_{2}\right)$ \\
\hline $\mathrm{I}-1$ & $\begin{array}{l}a(0,0)=h\left(L_{1}, L_{2}\right) \\
a\left(0, n_{2}\right)=2 h\left(L_{1}, L_{2}-n_{2}\right), n_{2}=1, \ldots, L_{2} \\
a\left(n_{1}, 0\right)=2 h\left(L_{1}-n_{1}, L_{2}\right), n_{1}=1, \ldots, L_{1} \\
a\left(n_{1}, n_{2}\right)=4 h\left(L_{1}-n_{1}, L_{2}-n_{2}\right), n_{1}=1, \ldots, L_{1}, n_{2}=1, \ldots, L_{2}\end{array}$ \\
\hline $\mathrm{I}-2$ & $\begin{array}{l}a\left(0, n_{2}\right)=2 h\left(L_{1}, L_{2}-n_{2}\right), n_{2}=1, \ldots, L_{2} \\
a\left(n_{1}, n_{2}\right)=4 h\left(L_{1}-n_{1}, L_{2}-n_{2}\right), n_{1}=1, \ldots, L_{1}, n_{2}=1, \ldots, L_{2}\end{array}$ \\
\hline $\mathrm{I}-3$ & $\begin{array}{l}a\left(n_{1}, 0\right)=2 h\left(L_{1}-n_{1}, L_{2}\right), n_{1}=1, \ldots, L_{1} \\
a\left(n_{1}, n_{2}\right)=4 h\left(L_{1}-n_{1}, L_{2}-n_{2}\right), n_{1}=1, \ldots, L_{1}, n_{2}=1, \ldots, L_{2}\end{array}$ \\
\hline $\mathrm{I}-4$ & $a\left(n_{1}, n_{2}\right)=4 h\left(L_{1}-n_{1}, L_{2}-n_{2}\right), n_{1}=1, \ldots, L_{1}, n_{2}=1, \ldots, L_{2}$ \\
\hline II-1 & $\begin{array}{l}h\left(n_{1}, L_{2}\right)=0, n_{1}=0, \ldots, N_{1}-1 \\
a\left(0, n_{2}\right)=2 h\left(L_{1}, L_{2}-n_{2}\right), n_{2}=1, \ldots, L_{2} \\
a\left(n_{1}, n_{2}\right)=4 h\left(L_{1}-n_{1}, L_{2}-n_{2}\right), n_{1}=1, \ldots, L_{1}, n_{2}=1, \ldots, L_{2}\end{array}$ \\
\hline II -2 & $\begin{array}{l}a\left(0, n_{2}\right)=2 h\left(L_{1}, L_{2}-n_{2}\right), n_{2}=1, \ldots, L_{2} \\
a\left(n_{1}, n_{2}\right)=4 h\left(L_{1}-n_{1}, L_{2}-n_{2}\right), n_{1}=1, \ldots, L_{1}, n_{2}=1, \ldots, L_{2}\end{array}$ \\
\hline II -3 & $\begin{array}{l}h\left(n_{1}, L_{2}\right)=0, n_{1}=0, \ldots, N_{1}-1 \\
a\left(n_{1}, n_{2}\right)=4 h\left(L_{1}-n_{1}, L_{2}-n_{2}\right), n_{1}=1, \ldots, L_{1}, n_{2}=1, \ldots, L_{2}\end{array}$ \\
\hline II -4 & $a\left(n_{1}, n_{2}\right)=4 h\left(L_{1}-n_{1}, L_{2}-n_{2}\right), n_{1}=1, \ldots, L_{1}, n_{2}=1, \ldots, L_{2}$ \\
\hline III-1 & $\begin{array}{l}h\left(L_{1}, n_{2}\right)=0, n_{2}=0, \ldots, N_{2}-1 \\
a\left(n_{1}, 0\right)=2 h\left(L_{1}-n_{1}, L_{2}\right), n_{1}=1, \ldots, L_{1} \\
a\left(n_{1}, n_{2}\right)=4 h\left(L_{1}-n_{1}, L_{2}-n_{2}\right), n_{1}=1, \ldots, L_{1}, n_{2}=1, \ldots, L_{2}\end{array}$ \\
\hline III-2 & $\begin{array}{l}h\left(L_{1}, n_{2}\right)=0, n_{2}=0, \ldots, N_{2}-1 \\
a\left(n_{1}, n_{2}\right)=4 h\left(L_{1}-n_{1}, L_{2}-n_{2}\right), n_{1}=1, \ldots, L_{1}, n_{2}=1, \ldots, L_{2}\end{array}$ \\
\hline III-3 & $\begin{array}{l}a\left(n_{1}, 0\right)=2 h\left(L_{1}-n_{1}, L_{2}\right), n_{1}=1, \ldots, L_{1} \\
a\left(n_{1}, n_{2}\right)=4 h\left(L_{1}-n_{1}, L_{2}-n_{2}\right), n_{1}=1, \ldots, L_{1}, n_{2}=1, \ldots, L_{2}\end{array}$ \\
\hline III-4 & $a\left(n_{1}, n_{2}\right)=4 h\left(L_{1}-n_{1}, L_{2}-n_{2}\right), n_{1}=1, \ldots, L_{1}, n_{2}=1, \ldots, L_{2}$ \\
\hline IV-1 & $\begin{array}{l}h\left(L_{1}, n_{2}\right)=0, n_{2}=0, \ldots, N_{2}-1 \\
h\left(n_{1}, L_{2}\right)=0, n_{1}=0, \ldots, N_{1}-1 \\
a\left(n_{1}, n_{2}\right)=4 h\left(L_{1}-n_{1}, L_{2}-n_{2}\right), n_{1}=1, \ldots, L_{1}, n_{2}=1, \ldots, L_{2}\end{array}$ \\
\hline VI-2 & $\begin{array}{l}h\left(L_{1}, n_{2}\right)=0, n_{2}=0, \ldots, N_{2}-1 \\
a\left(n_{1}, n_{2}\right)=4 h\left(L_{1}-n_{1}, L_{2}-n_{2}\right), n_{1}=1, \ldots, L_{1}, n_{2}=1, \ldots, L_{2}\end{array}$ \\
\hline IV-3 & $\begin{array}{l}h\left(n_{1}, L_{2}\right)=0, n_{1}=0, \ldots, N_{1}-1 \\
a\left(n_{1}, n_{2}\right)=4 h\left(L_{1}-n_{1}, L_{2}-n_{2}\right), n_{1}=1, \ldots, L_{1}, n_{2}=1, \ldots, L_{2}\end{array}$ \\
\hline IV-4 & $a\left(n_{1}, n_{2}\right)=4 h\left(L_{1}-n_{1}, L_{2}-n_{2}\right), n_{1}=1, \ldots, L_{1}, n_{2}=1, \ldots, L_{2}$ \\
\hline
\end{tabular}

Also, $\hat{H}_{\text {ee }}\left(\omega_{1}, \omega_{2}\right), \hat{H}_{\text {eo }}\left(\omega_{1}, \omega_{2}\right), \hat{H}_{\text {oe }}\left(\omega_{1}, \omega_{2}\right)$ and $\hat{H}_{\mathrm{oo}}\left(\omega_{1}, \omega_{2}\right)$ can be found from $\hat{H}\left(\omega_{1}, \omega_{2}\right)$ by

$$
\begin{aligned}
\hat{H}_{\mathrm{ee}}\left(\omega_{1}, \omega_{2}\right)= & \frac{1}{4}\left[\hat{H}\left(\omega_{1}, \omega_{2}\right)+\hat{H}\left(-\omega_{1}, \omega_{2}\right)\right. \\
& \left.+\hat{H}\left(\omega_{1},-\omega_{2}\right)+\hat{H}\left(-\omega_{1},-\omega_{2}\right)\right],
\end{aligned}
$$

$$
\begin{aligned}
\hat{H}_{\mathrm{eo}}\left(\omega_{1}, \omega_{2}\right)=\frac{1}{4}\left[\hat{H}\left(\omega_{1}, \omega_{2}\right)+\hat{H}\left(-\omega_{1}, \omega_{2}\right)\right. & \\
& \left.-\hat{H}\left(\omega_{1},-\omega_{2}\right)-\hat{H}\left(-\omega_{1},-\omega_{2}\right)\right], \\
\hat{H}_{\mathrm{oe}}\left(\omega_{1}, \omega_{2}\right)= & \frac{1}{4}\left[\hat{H}\left(\omega_{1}, \omega_{2}\right)-\hat{H}\left(-\omega_{1}, \omega_{2}\right)\right. \\
& \left.+\hat{H}\left(\omega_{1},-\omega_{2}\right)-\hat{H}\left(-\omega_{1},-\omega_{2}\right)\right]
\end{aligned}
$$


and

$$
\begin{aligned}
\hat{H}_{\mathrm{oo}}\left(\omega_{1}, \omega_{2}\right)= & \frac{1}{4}\left[\hat{H}\left(\omega_{1}, \omega_{2}\right)-\hat{H}\left(-\omega_{1}, \omega_{2}\right)\right. \\
& \left.-\hat{H}\left(\omega_{1},-\omega_{2}\right)+\hat{H}\left(-\omega_{1},-\omega_{2}\right)\right] .
\end{aligned}
$$

It is noted that $\hat{H}_{\mathrm{ee}}\left(\omega_{1}, \omega_{2}\right), \quad \hat{H}_{\mathrm{eo}}\left(\omega_{1}, \omega_{2}\right)$, $\hat{H}_{\text {oe }}\left(\omega_{1}, \omega_{2}\right)$ and $\hat{H}_{\text {oo }}\left(\omega_{1}, \omega_{2}\right)$ can be realized by Type I, II, III and IV 2-D sequences, respectively. Hence given a desired magnitude response, we can realize it by either a single type 2-D sequence or several mixed type 2-D sequences. In this paper, the linear-phase 2-D FIR filters are divided into three classes as below.

Quadrantal-plane filter: The filters which can be realized by only a single type of $2-D$ sequence.

Half-plane filter: The filters which can be realized by synthesizing two types of 2-D sequences.

Full-plane filter: The filters which can be realized by synthesizing three or four types of 2-D sequences.

In the remainder of this paper, we will focus on the applications of four major types of 2-D sequences for designing linear-phase 2-D FIR digital filters using the eigen-filter approach, which has successfully been used to design linear-phase 1-D filters and quadrantally symmetric 2-D filters [2-4].

\section{Design of quadrantal-plane linear-phase 2-D FIR filters}

When the desired frequency response, by excluding the linear-phase part, is a real-valued function, it can be realized by Type I or Type IV 2-D sequences. On the contrary, if the desired frequency response is an imaginary-valued function, Type II and Type III 2-D sequences are suitable for these designs. For example, the typical 2-D circular filters and fan-type filters can be realized by Type I 2-D sequences, which has been discussed in [4].

Example 1. In this example, we want to design a filter with the desired magnitude response

$D\left(\omega_{1}, \omega_{2}\right)= \begin{cases}1, & \omega_{1} \cdot \omega_{2}>0, \\ -1 & \omega_{1} \cdot \omega_{2}<0 .\end{cases}$

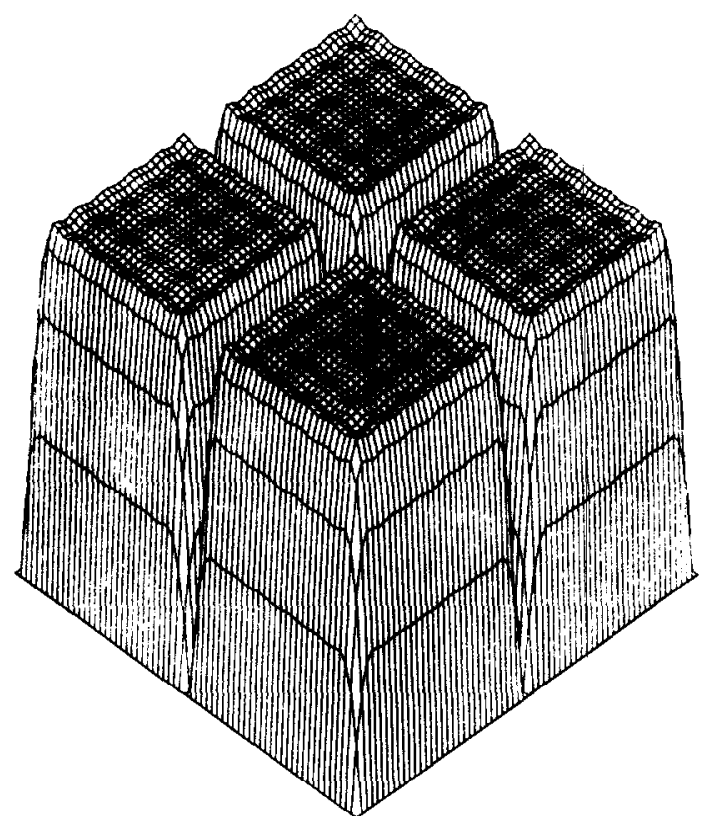

Fig. 1. Example 1: Amplitude response of a $23 \times 232$-D lincar-phase filter designed by a Type IV-1 2-D sequence.

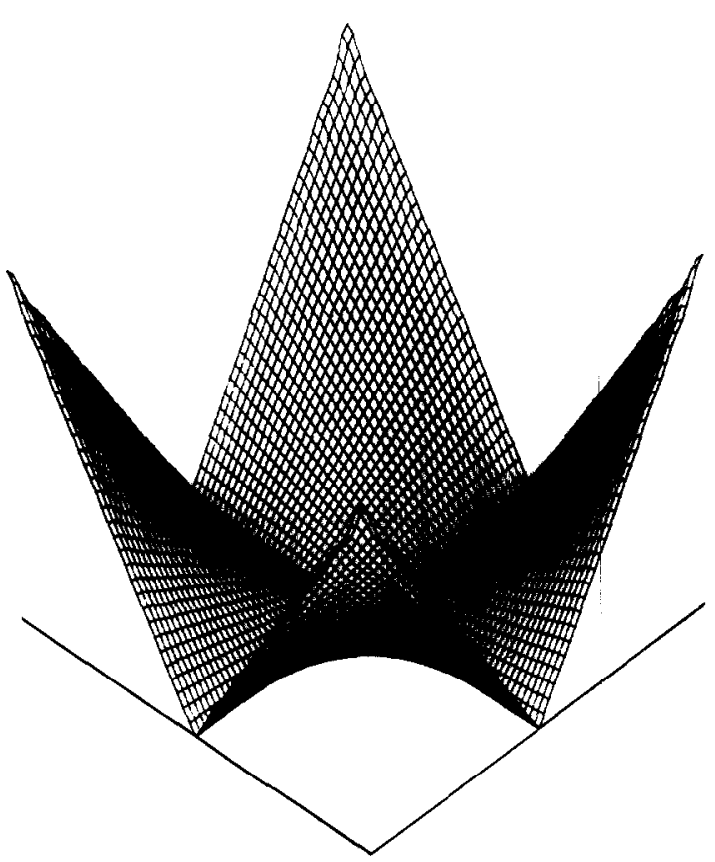

Fig. 2. Example 2: Amplitude response of a $27 \times 262$ 2-D linear-phase filter designed by a Type II-2 2-D sequence. 
Here, we use a $23 \times 23$ Type IV-1 2-D sequence to design it, and the eigenfilter approach in [4] can be applied after slight modification. The resultant amplitude response is shown in Fig. 1 in which the cutoff frequencies are $0.1 \pi$ and $0.9 \pi$ in both the $\omega_{1}$ and $\omega_{2}$-axis.

Example 2. A third-order partial differentiator is designed in this example. Excluding the part of linear phase, the desired frequency response is

$D\left(\omega_{1}, \omega_{2}\right)=-\mathrm{j} \omega_{1}^{2} \omega_{2}, \quad-\pi \leqslant \omega_{1}, \omega_{2} \leqslant \pi$.

When a $27 \times 26$ Type II-2 2-D sequence is used, the amplitude response is shown in Fig. 2.

\section{Design of half- and full-plane linear-phase 2-D FIR filters}

The half-plane filters can be divided into two categories, i.e. 'non-neighbor symmetric/antisymmetric half-plane filters' and 'neighbor symmetric/antisymmetric half-plane filters'. Four typical half-plane filter examples are given in Fig. 3. Fig. 3(a) presents a non-neighbor symmetric halfplane filter, and the realized sequence is organized by a Type I sequence and a Type IV sequence. The example of a non-neighbor antisymmetric halfplane filter is shown in Fig. 3(b) which can be implemented by combining a Type II sequence and a Type III sequence, so the filter coefficients are pure imaginary. Fig. 3(c) presents the example of a neighbor symmetric half-plane filter, and Type I sequence plus a Type III sequence can realize it, so the filter coefficients are complex. Fig. 3(d) shows a neighbor antisymmetric half-plane filter which can be implemented by using a Type II sequence and a Type IV sequence, hence the filter coefficients are also complex.

Example 3 (Design of a non-neighbor symmetric half-plane filter). This example deals with the design of a filter with the desired magnitude response $D\left(\omega_{1}, \omega_{2}\right)= \begin{cases}1, & \omega_{1} \cdot \omega_{2}>0 \\ 0, & \omega_{1} \cdot \omega_{2}<0 .\end{cases}$

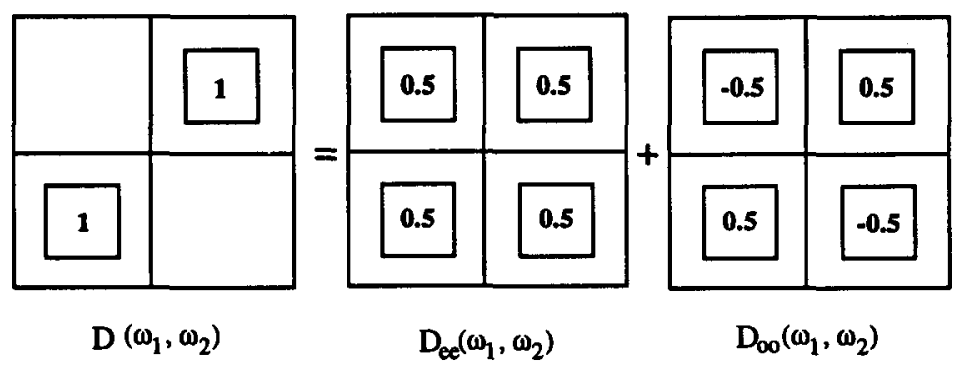

(a)

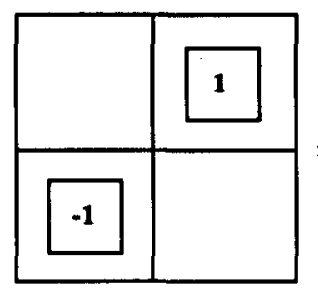

$D\left(\omega_{1}, \omega_{2}\right)$

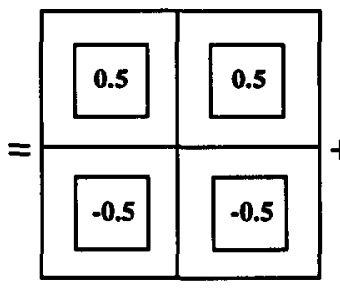

$\mathrm{D}_{\mathrm{eo}}\left(\omega_{1}, \omega_{2}\right)$

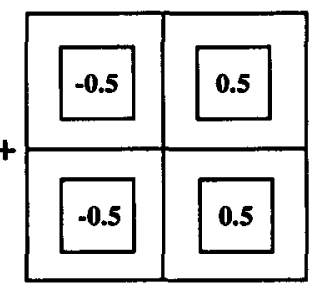

$\mathrm{Doe}\left(\omega_{1}, \omega_{2}\right)$

(b)

Fig. 3. Illustrated examples to explain the type of half-plane 2-D filters: (a) non-neighbor symmetric half-plane filter, (b) non-neighbor antisymmetric half-plane filter. 


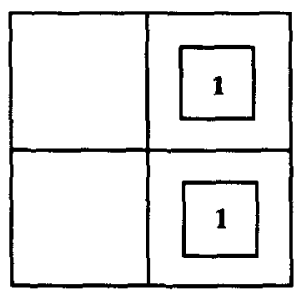

$D\left(\omega_{1}, \omega_{2}\right)$

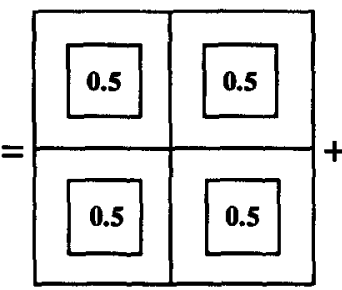

$D_{\mathrm{ee}}\left(\omega_{1}, \omega_{2}\right)$

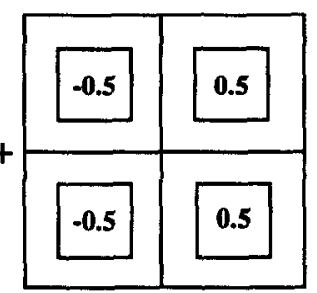

$\operatorname{Doe}\left(\omega_{1}, \omega_{2}\right)$

(c)
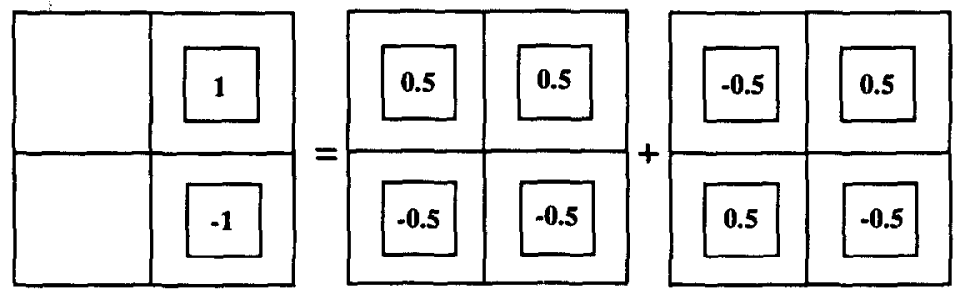

$\mathrm{D}\left(\omega_{1}, \omega_{2}\right)$

$D_{\mathrm{eo}}\left(\omega_{1}, \omega_{2}\right)$

$\mathrm{D}_{\mathrm{oo}}\left(\omega_{1}, \omega_{2}\right)$

(d)

Fig. 3. (c) Neighbor symmetric half-plane filter, (d) neighbor antisymmetric half-plane filter.

By Eq. (29), $D\left(\omega_{1}, \omega_{2}\right)$ can be obtained by summing the four parts as

$$
\begin{aligned}
D\left(\omega_{1}, \omega_{2}\right)= & D_{\mathrm{ee}}\left(\omega_{1}, \omega_{2}\right)+D_{\mathrm{eo}}\left(\omega_{1}, \omega_{2}\right) \\
& +D_{\mathrm{oe}}\left(\omega_{1}, \omega_{2}\right)+D_{\mathrm{oo}}\left(\omega_{1}, \omega_{2}\right),
\end{aligned}
$$

and from (34)-(37) we can get

$D_{\text {ee }}\left(\omega_{1}, \omega_{2}\right)=0.5$,

$D_{\mathrm{co}}\left(\omega_{1}, \omega_{2}\right)=D_{\mathrm{oe}}\left(\omega_{1}, \omega_{2}\right)=0$

and

$D_{\mathrm{oo}}\left(\omega_{1}, \omega_{2}\right)= \begin{cases}0.5, & \omega_{1} \cdot \omega_{2}>0 \\ -0.5, & \omega_{1} \cdot \omega_{2}<0\end{cases}$

Hence, two types of 2-D sequences are needed, i.e. Type I sequence realizes $D_{\text {ee }}$ and Type IV sequence realizes $D_{\mathrm{oo}}$. Since $D_{\mathrm{ee}}\left(\omega_{1}, \omega_{2}\right)$ corresponds to a unit impulse response in the spatial domain and $D_{\text {oo }}\left(\omega_{1}, \omega_{2}\right)$ is similar to Example 1, we only add a unit impulse to the origin of the resultant impulse response in Example 1, and then normalize the amplitude response to unit. The overall amplitude response is shown in Fig. 4.

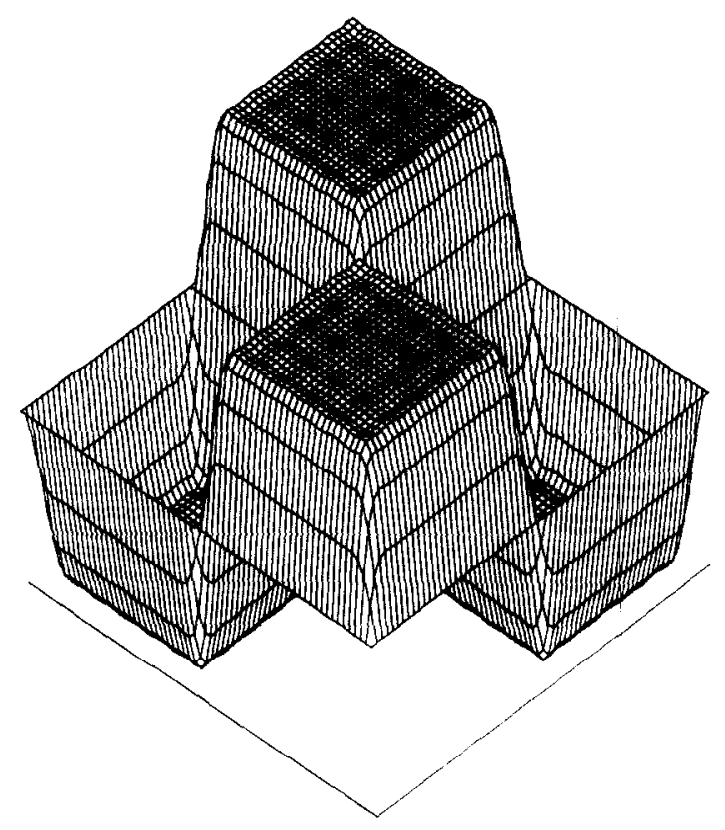

Fig. 4. Example 3: Amplitude response of a $23 \times 23$ non-neighbor symmetric half-plane filter. 

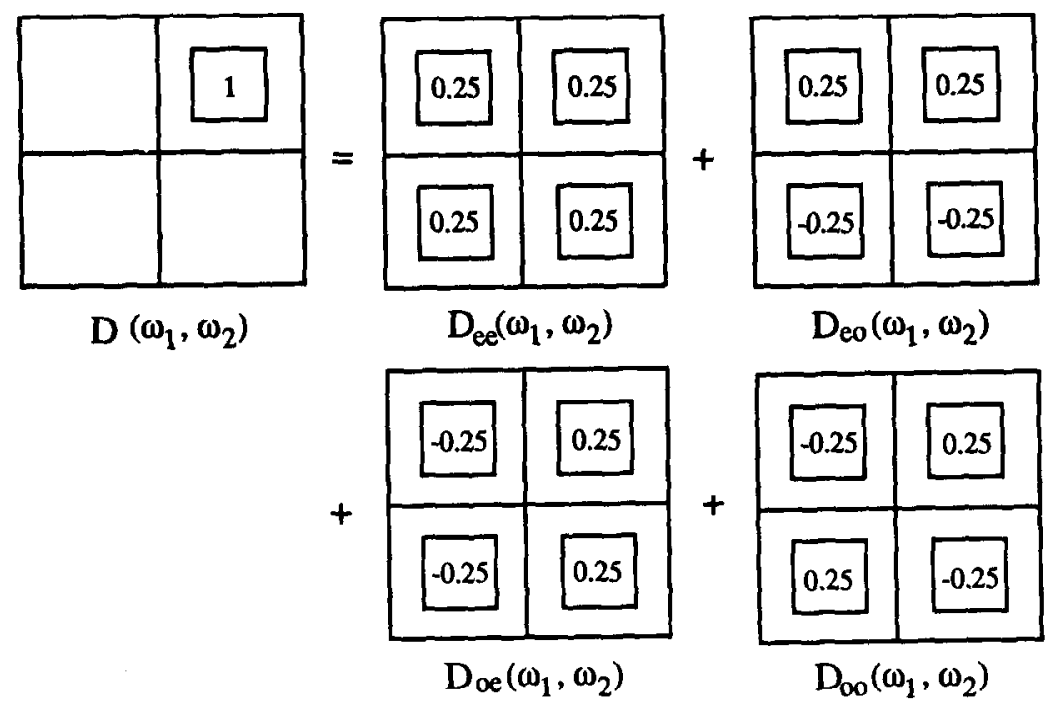

(a)

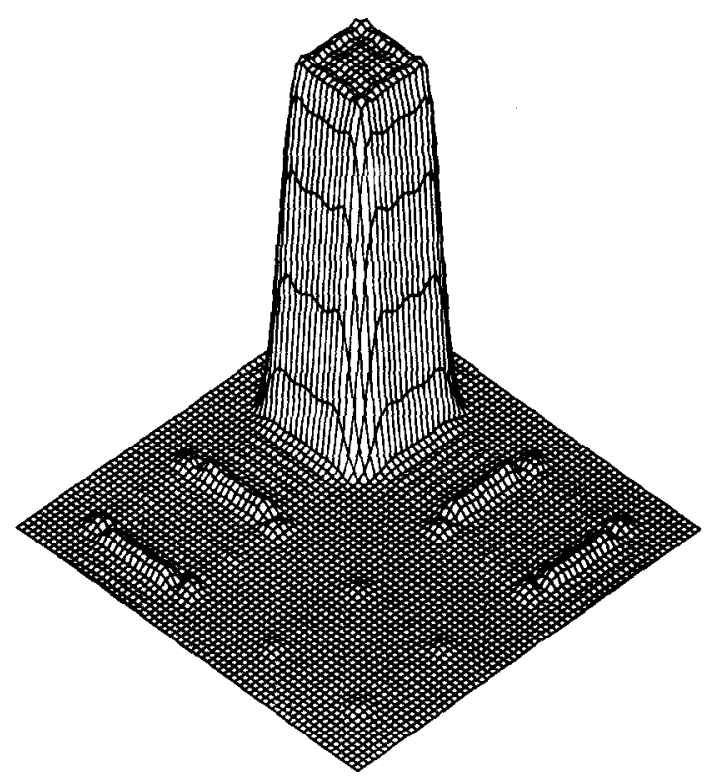

(b)

Fig. 5. Example 4: (a) Desired amplitude response and its ingredients, (b) the actual amplitude response. 
As to the design of full-plane filters, the four types of 2-D sequences are required. For simplicity, an example is presented to demonstrate the design procedures.

Example 4. This example presents the design of a full-plane filter with the desired response and its ingredients as shown in Fig. $5(\mathrm{a}) . D_{\mathrm{ee}}$ and $D_{\mathrm{oo}}$ can be synthesized by using Type I and Type IV 2-D sequences, respectively, and $D_{\text {eo }}$ and $D_{\text {oe }}$ can be approached by using Type II and Type III 2-D sequences. Fig. 5(b) shows the resultant amplitude response with filter length $27 \times 27$, if the above synthetic method is used.

\section{Conclusions}

In this paper, we have presented the symmetric properties of 2-D sequences and their applications for designing linear-phase 2-D FIR filters. It is shown that there are 16 types of cases to be considered according to the symmetry/antisymmetry of 2-D sequences in both directions and their filter lengths (even or odd). The corresponding types of amplitude responses are tabulated into a complete table if these 2-D sequences are used to realize 2-D filters. Also, the definitions of quadrantal-plane, half-plane and full-plane filters are described along with numerical examples designed by the eigenfilter approach.

\section{References}

[1] L.R. Rabiner and B. Gold, Theory and Application of Digital Signal Processing, Prentice-Hall, Englewood Cliffs, NJ, 1975.

[2] S.C. Pei and J.J. Shyu, "Eigenfilter design of higher order digital differentiators", IEEE Trans. Acoust. Speech Signal Process., Vol. 37, April 1989, pp. 505-511.

[3] P.P. Vaidyanathan and T.Q. Nguyen, "Eigenfilter: A new approach to least-squares FIR filter design and applications including Nyquist filters", IEEE Trans. Circuits Systems, Vol. 34, January 1987, pp. 11-23.

[4] S.C. Pei and J.J. Shyu, "2-D FIR eigenfilters: A least squares approach", IEEE Trans. Circuits Systems, Vol. 37, January 1990 , pp. 24-34. 